\title{
The interperiosteodural concept applied to the jugular foramen and its compartmentalization
}

\author{
Florian Bernard, MD, 1 llyess Zemmoura, MD, PhD, ${ }^{2-4}$ Jean Philippe Cottier, MD, PhD, ${ }^{2,5}$ \\ Henri-Dominique Fournier, MD, PhD, ${ }^{1,6}$ Louis-Marie Terrier, MD, MSc, ${ }^{2-4}$ and \\ Stéphane Velut, MD, $\mathrm{PhD}^{2-4}$
}

\begin{abstract}
${ }^{1}$ Department of Neurosurgery, CHU Angers; ${ }^{2}$ Université François-Rabelais de Tours, Inserm, Imagerie et Cerveau, UMR U930, Tours; ${ }^{3}$ Anatomy Laboratory, Faculté de Médecine, Tours; Departments of ${ }^{4}$ Neurosurgery and ${ }^{5}$ Neuroradiology, CHRU de Tours; and ${ }^{6}$ Anatomy Laboratory, Faculté de Médecine, Angers, France
\end{abstract}

OBJECTIVE The dura mater is made of 2 layers: the endosteal layer (outer layer), which is firmly attached to the bone, and the meningeal layer (inner layer), which directly covers the brain and spinal cord. These 2 dural layers join together in most parts of the skull base and cranial convexity, and separate into the orbital and perisellar compartments or into the spinal epidural space to form the extradural neural axis compartment (EDNAC). The EDNAC contains fat and/or venous blood. The aim of this dissection study was to anatomically verify the concept of the EDNAC by focusing on the dural layers surrounding the jugular foramen area.

METHODS The authors injected 10 cadaveric heads ( 20 jugular foramina) with colored latex and fixed them in formalin. The brainstem and cerebellum of 7 specimens were cautiously removed to allow a superior approach to the jugular foramen. Special attention was paid to the meningeal architecture of the jugular foramen, the petrosal inferior sinus and its venous confluence with the sigmoid sinus, and the glossopharyngeal, vagus, and accessory nerves. The 3 remaining heads were bleached with a $20 \%$ hydrogen peroxide solution. This procedure produced softening of the bone without modifying the fixed soft tissues, thus permitting coronal and axial dissections.

RESULTS The EDNAC of the jugular foramen was limited by the endosteal and meningeal layers and contained venous blood. These 2 dural layers joined together at the level of the petrous and occipital bones and separated at the inferior petrosal sinus and the sigmoid sinus, and around the lower cranial nerves, to form the EDNAC. Study of the dural sheaths allowed the authors to describe an original compartmentalization of the jugular foramen in 3 parts: 2 neural compartments-glossopharyngeal and vagal—and the interperiosteodural compartment.

CONCLUSIONS In this dissection study, the existence of the EDNAC concept in the jugular foramen was demonstrated, leading to the proposal of a novel 3-part compartmentalization, challenging the classical 2-part compartmentalization, of the jugular foramen.

https://thejns.org/doi/abs/10.3171/2017.1.JNS161890

KEY WORDS jugular foramen; anatomy; interperiosteodural concept; skull base surgery

$\mathrm{T}$ HE jugular foramen $(\mathrm{JF})$ is one of the more complex skull base foramina. Viewed from inside the cranium, its shape mimics a teardrop, with a narrow anteromedial part and an enlarged posterolateral margin. $2,8,12,13$ The structures that cross the JF are the sigmoid sinus (SS), the jugular bulb, the inferior petrosal sinus (IPS), meningeal branches of the ascending pharyngeal and occipital arteries, and lower cranial nerves-the glossopharyngeal nerve (cranial nerve [CN] IX), vagus nerve
(CN X), and accessory nerve (CN XI). ${ }^{14,15}$ The complexity of the JF is due to modifications of the neurovascular relationships, from the cerebral posterior fossa to the parapharyngeal space. Thus, the surgical approach to tumors of the JF requires a particularly comprehensive knowledge of anatomy, especially that of its dura mater.

The meninges enclosing the central nervous system have generally been considered to consist of 3 different macroscopic layers: the outermost layer (dura mater), the

ABBREVIATIONS CN = cranial nerve; EDNAC = extradural neural axis compartment; EL = endosteal layer; ICA = internal carotid artery; IL = intrajugular ligament; IPS = inferior petrosal sinus; $\mathrm{JF}=$ jugular foramen; $\mathrm{ML}=$ middle layer; $\mathrm{SS}=$ sigmoid sinus.

SUBMITTED July 26, 2016. ACCEPTED January 5, 2017.

INCLUDE WHEN CITING Published online September 8, 2017; DOI: 10.3171/2017.1.JNS161890. 
intermediate layer (arachnoid mater), and the innermost layer (pia mater). The dura mater forms the outer meninx and covers the spine and the brain. The works of Tap$\operatorname{tas}^{18-20}$ on the cavernous sinus significantly improved the knowledge of the descriptive anatomy of the dura mater. Subsequently, Parkinson ${ }^{13}$ described an anatomical concept, the extradural neural axis compartment (EDNAC), extending from the coccyx to the orbit.

According to the EDNAC concept, the dura mater lining the internal surface of the calvaria is formed by 2 layers: the outer layer (periosteum or the so-called endosteal layer [EL]), which is thin and strongly attached to the bone, and the inner (meningeal) layer (ML), which is thick and directly covers the brain. ${ }^{7,13}$ The ML and EL are closely adherent in most parts of the skull base and convexity. Nevertheless, the ML and EL separate in some regions, as in the orbit or the spinal epidural space, leading to the formation of an interperiosteodural space, containing veins and/or fat tissue. Destrieux et al. ${ }^{5}$ reported that the petroclival venous confluence was located between these 2 layers of dura mater, and the existence of this space was also demonstrated in the perisellar area and verified with electron microscopy by François et al. ${ }^{7,8}$

The aim of the present study was to rethink the compartmentalization of the JF, by focusing on the interperiosteodural concept, using microanatomical cadaver dissections.

\section{Methods}

\section{Specimen Preparation}

We studied 10 adult cadaveric heads obtained from the body donation program of our institution. We injected the blood vessels with dyed neoprene latex, using red ink for the internal carotid artery (ICA) and vertebral arteries, and blue ink for the jugular veins. All of the specimens were then fixed with a $10 \%$ formalin solution. Three of the 10 cadaveric heads were subsequently soaked in a $20 \%$ hydrogen peroxide solution for several weeks. This process softened the bone without modifying the fixed, soft tissues, thus allowing the bone to be cut with an ordinary scalpel, thereby permitting coronal and axial dissections. Careful removal of the calvaria and of the brain allowed us to use a superior approach to the JF.

\section{Dissection Method}

The petrous bone was drilled to expose the dura mater in the superior part of the JF. Coronal and axial sections were then performed. The dural layers were progressively dissected under optic magnification (250-, 300-, and 400$\mathrm{mm}$ focal distances) with a surgical microscope (Leica M720 OH 5).

\section{High-Resolution Photographs}

Silver halide photographs were obtained with a Hasselblad camera and lenses (Victor Hasselblad AB) and Fujichrome Velvia 50 color reversal film (Fujifilm).

\section{Results}

The JF was located at the posterior aspect of the petrooccipital fissure. It was bounded anterolaterally by the pe- trous bone and posteromedially by the basilar part of the occipital bone. The long axis of the foramen was directed from posterolateral to anteromedial. The JF did not correspond to a simple hole but to a canal, with a horizontal portion lying over the jugular process of the occipital bone and a vertical portion covered by the jugular fossa of the temporal bone. From an intracranial viewpoint, its shape mimicked a teardrop with a narrow anteromedial part and an enlarged posterolateral margin (Fig. 1A). The structures that traversed the JF were the SS, jugular bulb, IPS, meningeal branches of the ascending pharyngeal and occipital arteries, and lower cranial nerves.

\section{Neural Content of the JF}

During their course from the brainstem to the upper neck, CN IX, CN X, and CN XI ran from a medial and horizontal trajectory to a lateral and vertical one (Figs. $1 \mathrm{~B}$ and 2A). Relationships between these 3 lower cranial nerves also moved from a craniocaudal configuration in their cisternal course (Figs. 1A, 2B, and 3B) to an anteroposterior one in the neck. These changes occurred at the level of the JF, where the lower cranial nerves converge. The shape of the jugular tubercle and the occipital condyle that represents the floor of the JF explained and allowed these reroutings (Figs. 1B and 2A). The cranial course of the lower cranial nerves can be thus classified into 3 segments: preforaminal or cisternal, intraforaminal, and extraforaminal or cervical. This work did not concern the extraforaminal course.

\section{Cisternal Course}

The vagus nerve $(\mathrm{CN} \mathrm{X})$ arose from the upper medulla dorsal to the retro-olivary sulcus. This nerve coursed along the caudal aspect of the choroid plexus at the lateral recess of the fourth ventricle, then passed through the inferior cerebellopontine cistern and the anteromedial perforation of the ML of the JF (Fig. 3A). CN X consisted of an average of 8 fiber bundles (range 4-15), which exited posteriorly at the retro-olivary sulcus below CN IX (Fig. 4B). CN XI displayed distinct cranial and spinal rootlets. A mean of 11 cranial rootlets (range 6-16) arose below $\mathrm{CN} \mathrm{X}$, at the lower level of the medulla oblongata, while the spinal ones arose behind the first digitations of the dentate ligament, from the funiculus lateralis of the upper cervical cord.

Approaching the nervous porus of the JF, $\mathrm{CN} \mathrm{X}$ and $\mathrm{CN}$ XI rootlets converged and penetrated the same dural hole, corresponding to a cribriform perforation of the $\mathrm{ML}$ (Figs. 1A, 4, 5A, and 6B).

\section{Intraforaminal Course}

CN IX, CN X, and CN XI entered the JF and briefly coursed horizontally before turning at a right angle and taking a vertical course (Figs. 1B, 2A, 3A, and 5B). Thereafter, CN IX turned sharply $\left(90^{\circ}\right)$ inferiorly and then coursed along the medial side of the intrajugular ridge (Figs. 1B and 2A). Otherwise, CN X and CN XI turned along the jugular tubercle, demonstrating a softer rerouting. Moreover, the horizontal part of CN IX was longer than that of $\mathrm{CN}$ X or CN XI. 

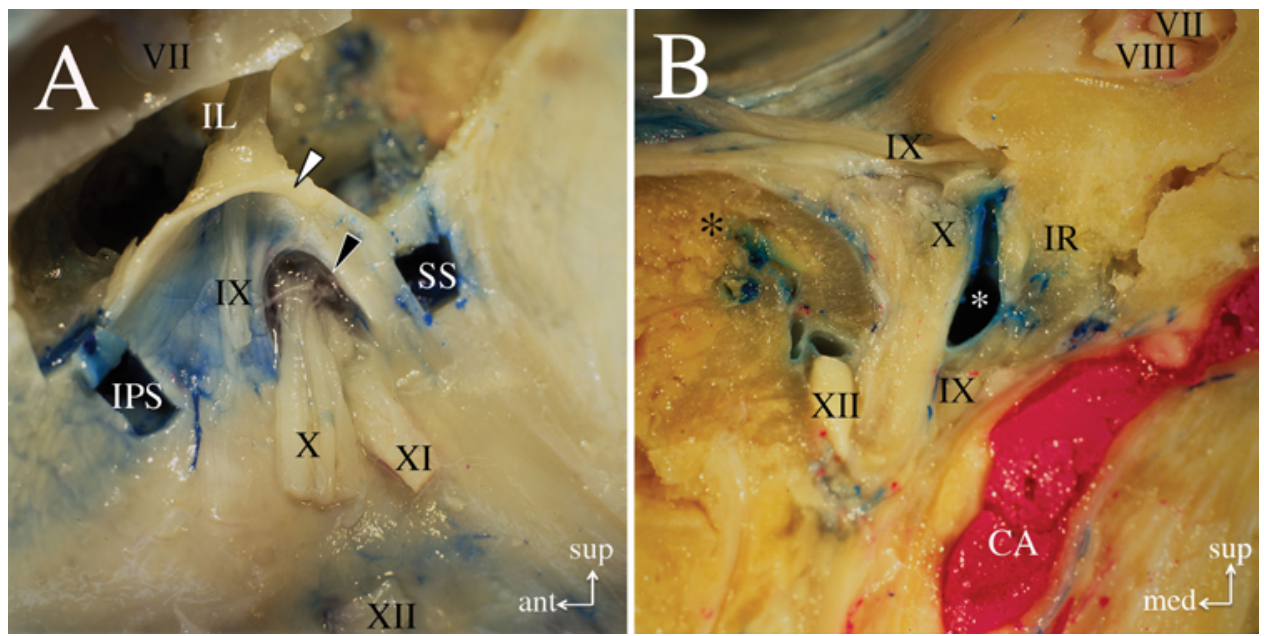

FIG. 1. A: Medial view of a right JF, after petrosal drilling and removal of the intrajugular process, showing the intraforaminal dura. The JF's shape mimics a teardrop, with a narrow anteromedial part and an enlarged posterolateral margin. The dural sheath of CN $X-X I$ was found caudal to the dural sheath of CN IX. The ML, which covers the petro-occipital fissure, forms an inferior ring within which CNs X and XI pass (black arrowhead). CN IX passes through the superior ring (white arrowhead). B: Coronal section of a right JF through $\mathrm{CN} X$. The intrajugular ridge (IR) is lateral and posterior to $\mathrm{CN} I X$, and lateral and superior to CN X. The shape of the jugular tubercle of the occipital bone (black asterisk) explains and allows the rerouting of the lower cranial nerves. The IPS and SS communicate within an interperiosteodural space between CN IX and CN X (white asterisk). CN XII is located inferior and medial to the JF. ant = anterior; $\mathrm{CA}=$ internal carotid artery; IL = intrajugular ligament; IPS = inferior petrosal sinus; IX = CN IX; med = medial; SS = sigmoid sinus; sup = superior; VII = CN VII; VIII = CN VIII; X = CN X; XI = CN XI; XII = CN XII.

The hypoglossal canal does not belong to the JF but is in close proximity. The hypoglossal nerve (CN XII) exited the medulla oblongata at the level of the preolivary sulcus and coursed anterolaterally to enter the hypoglossal foramen. It then coursed through the hypoglossal canal, which was located inferiorly and medially to the JF and inferiorly to the jugular tubercle and the superior aspect of the medial part of the occipital condyle. On a coronal section, it was possible to demonstrate that CN XII was in close proximity to the other lower cranial nerves (Fig. 1B).

\section{Venous Relationships}

The sigmoid sinus (SS) drained into the posterior aspect of the jugular bulb (Fig. 6). The jugular bulb varied considerably in size and shape from one specimen to an-
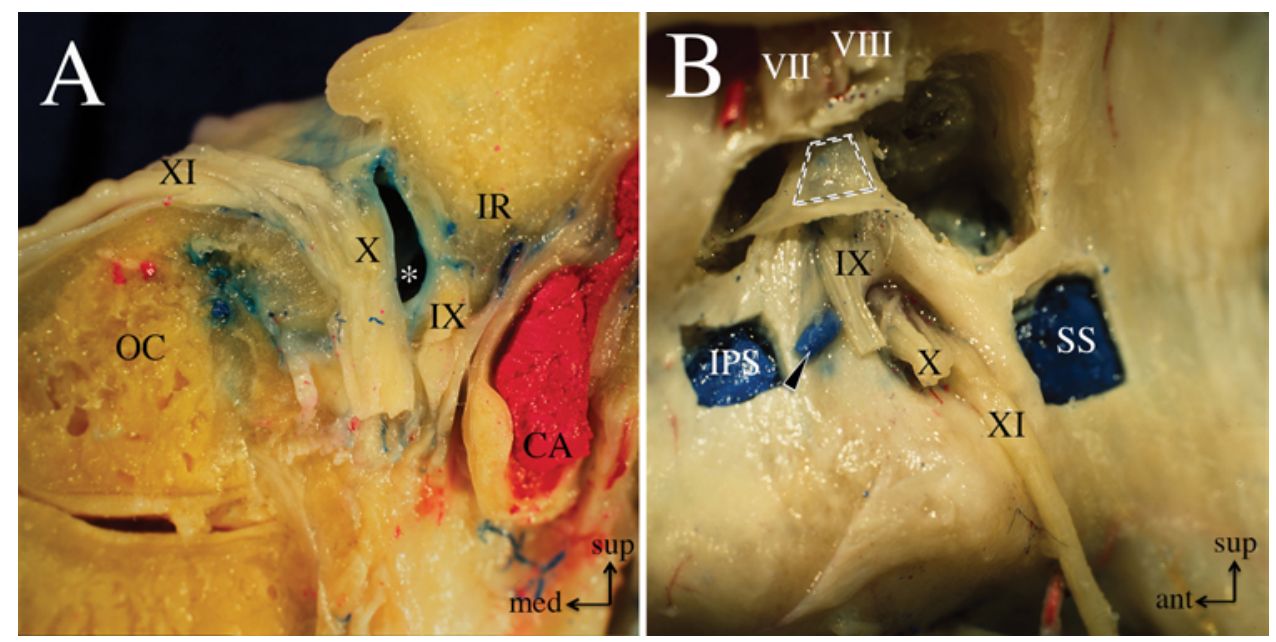

FIG. 2. A: Coronal section through $\mathrm{CNs} X$ and $\mathrm{XI}$ of the right JF. Note the proximity of the internal carotid artery to CN IX. During their course the lower cranial nerves run from a medial and horizontal trajectory to a lateral and vertical one. The white asterisk indicates the interperiosteodural space, which allows a venous communication between the IPS and SS. B: Medial view of a right $\mathrm{JF}$, after petrosal drilling and removal of the intrajugular process, showing the intraforaminal dura without removal of the superior dural sheath of CN IX (broken white lines). Although the diameter of the IPS is variable, no interperiosteodural space is found at the anterocranial part of the porus of CN IX. In this specimen, an emissary jugular vein (black arrowhead) joins the posterior end of the IPS, caudal to CN IX. OC = occipital condyle. 

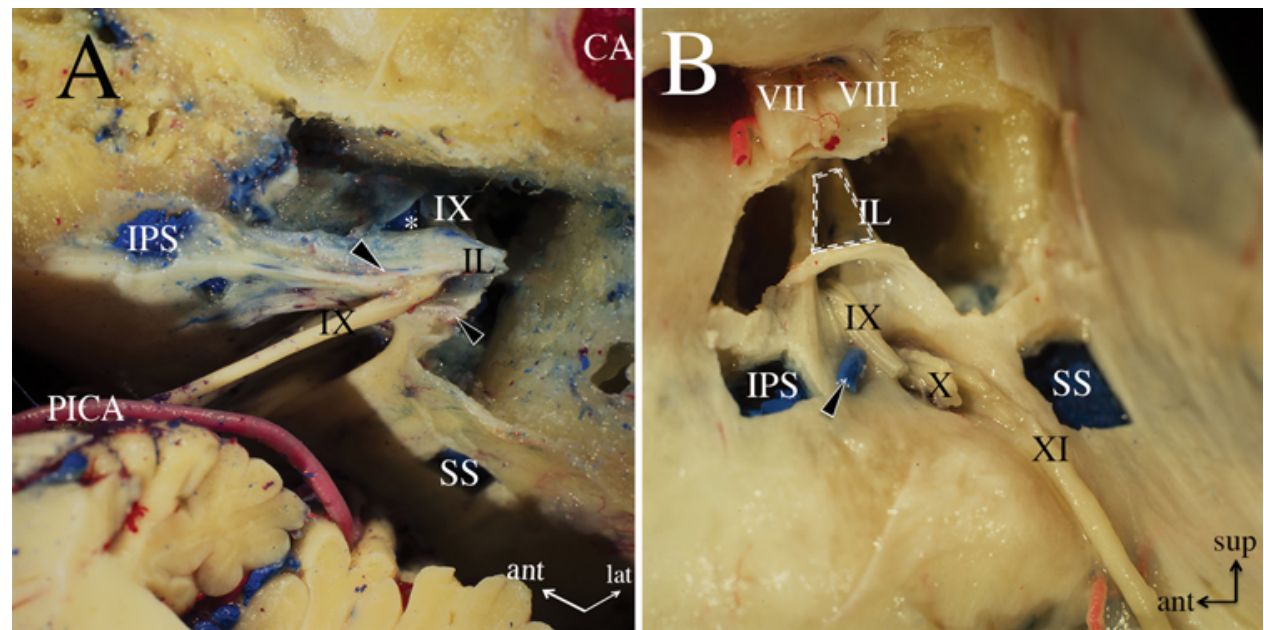

FIG. 3. A: Axial section of a right JF, obtained after petrosal drilling and removal of the intrajugular process, showing the intraforaminal dura. Two thickenings of the ML (black arrowheads) begin intracranially and continue within the JF, reaching up to a point laterally and constituting what we have referred to as the intrajugular ligament (IL). Communication between the IPS and SS in an interperiosteodural space is indicated by the white asterisk. B: Superior view of the same right JF as in Fig. 2B, after petrosal drilling and removal of the intrajugular process, showing the intraforaminal dura after removal of the superior dural sheath of CN IX (broken white lines), exposing the IL. The black arrowhead indicates an emissary vein coming from the IPS. lat = lateral; PICA = posterior inferior cerebellar artery.

other. The SS was the largest source of venous drainage into the JF. This sinus descended vertically along the sigmoid sulcus and then turned anteriorly toward the JF (Fig. 4A). At the level of the sinusojugular crest of the occipital bone, the SS took an S shape and continued its course ventrally to the petrous bone to form the jugular bulb.

The IPS linked the cavernous and basilar sinuses with the jugular bulb. It traveled along the petroclival fissure inside an interperiosteodural space (Fig. 4A). It then coursed between the dural sheaths of CN IX and CNs X-XI, i.e., inferior to CN IX and superior to CNs X and XI (Fig. 1B). In 4 specimens, we observed an interperiosteodural single connection between the SS and IPS inferiorly to the CNs $\mathrm{X}$ and XI (Fig. 6A).

\section{The Dura of the JF}

The endocranial wall of the edge of the JF was formed by the apposition of 2 meningeal layers (Fig. 4A): the first was an expansion of the EL covering the bones of the posterior cranial fossa, and the second was the ML proper. The inner layer overlying the venous jugular compartment contained 2 perforations, both of which were medial to the intrajugular processes. One was the glossopharyngeal
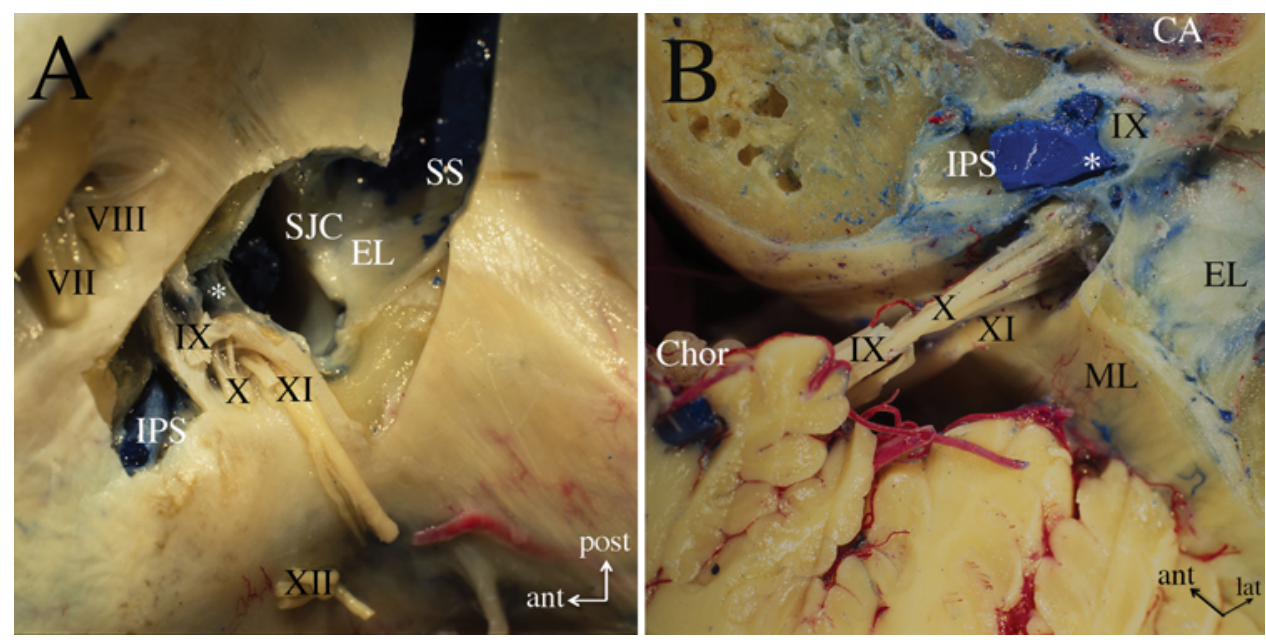

FIG. 4. A: Superomedial view of a right JF, after removal of the ML of the dura and blue latex in the SS, showing the floor of the SS (EL) along the sinusojugular crest (SJC) of the occipital bone. The IPS and SS communicate through an interperiosteodural space (white asterisk) located between the CN IX dural sheath superiorly and the CN X-XI dural sheath inferiorly. B: Axial section of a right JF through CN IX. The interperiosteodural space allowing venous communication between the IPS and SS is indicated by the white asterisk and is located between the CN IX dural sheath superiorly and the CN X-XI dural sheath inferiorly. Chor = choroid plexus; post = posterior. 

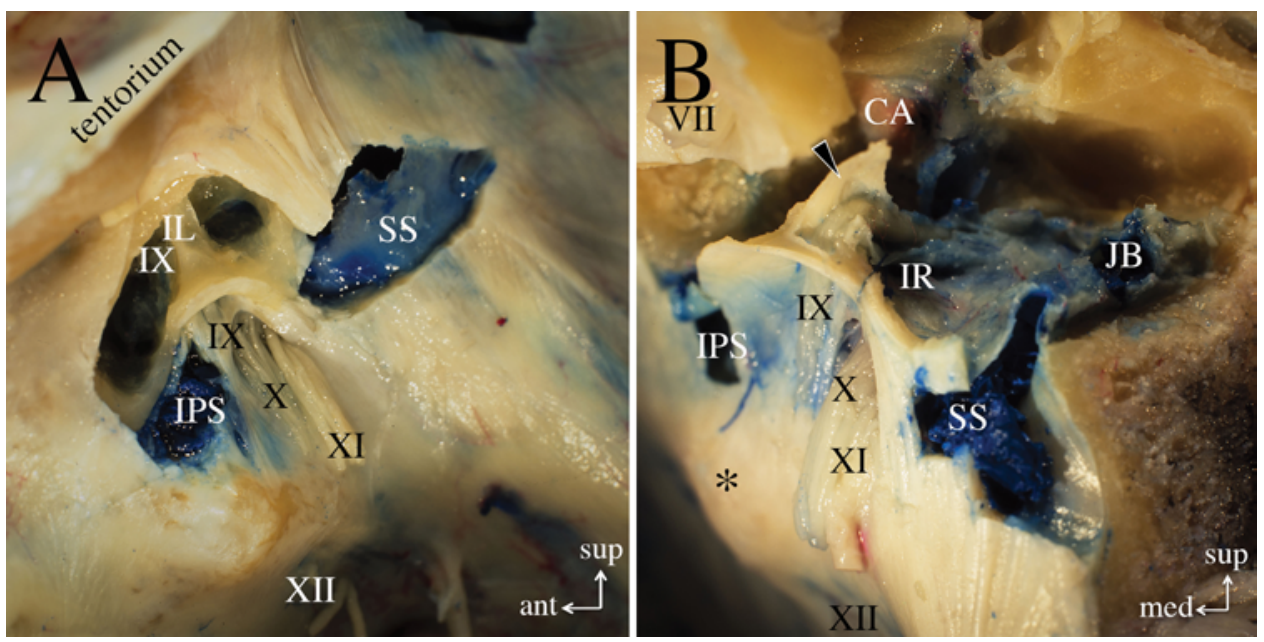

FIG. 5. A: Superomedial view of a right JF, after petrosal drilling and removal of the intrajugular process and the tentorium, showing the intraforaminal periosteal dura. B: Posteromedial view of a right JF. During their course, the lower cranial nerves run from a medial and horizontal trajectory to a lateral and vertical one. In all specimens, the ML anterior to CN IX and the ML posterior to CN IX form an IL, directed upward and laterally (black arrowhead). The posterior part of the IL is resected, showing the intraforaminal course of the CN IX. The intrajugular ridge (IR) is removed. Compared with CNs X and XI, CN IX courses in a distinct dural sheath. The black asterisk indicates the jugular tubercle. JB = jugular bulb.

meatus, through which CN IX passed. The other one was the vagal meatus, through which CNs $\mathrm{X}$ and $\mathrm{XI}$ passed (Figs. 1A, 2B, 3B, 4A, and 5).

The floor and the roof of the JF (Fig. 4A) and the roof of the jugular bulb (Fig. 6B) were formed by only the EL. In contrast, the medial wall of the SS and IPS was formed by the ML (Fig. 1A). The ML folded so that it overlaid the meatus of CNs IX, X, and XI and described 2 dural rings. $\mathrm{CN}$ IX reached the superior ring, while CNs X and XI reached the inferior ring (Fig. 1A).

At the level of their porus, the dural sheaths of $\mathrm{CNs}$ IX-XI were also composed of 2 layers. But at the level of their intraforaminal course, distinguishing between EL and ML was challenging. The ML that composed the porus of CN IX was thick (Fig. 2B). The superior wall of the dural sheath of the intraforaminal horizontal part of $\mathrm{CN}$ IX was composed of the 2 layers stuck together. Indeed, the EL covered its petrosal roof, while the ML appeared inferior to the EL. At this level, we identified 2 thickenings of the ML, which began intracranially (Fig. 3B): a posterior thickening immediately close to the vagal porus, separating CN IX from the petrous bone and the SS, and an anterior thickening, separating CN IX from the petrous bone. Both thickenings continued within the JF, reaching up to a lateral point and forming, respectively, the anterior and posterior edges of the superior dural sheath of CN IX.
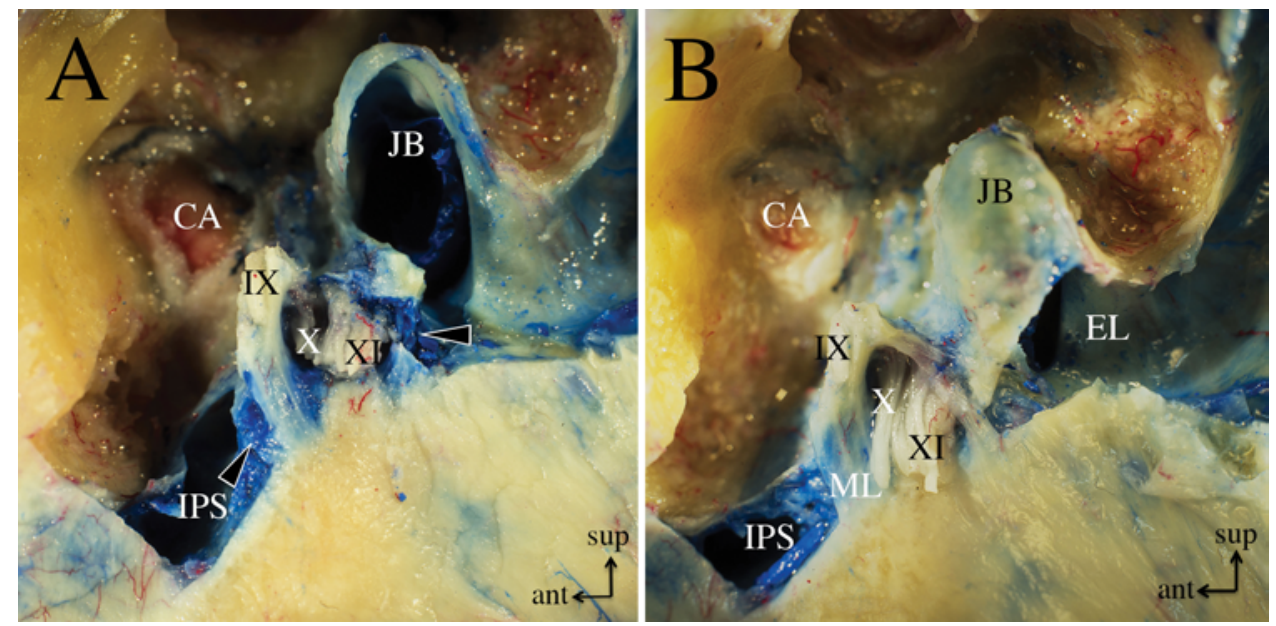

FIG. 6. A: Superomedial view of a right JF after petrosal drilling and removal of the intrajugular process, the dural sheath of $\mathrm{CN}$ IX, and the meningeal layer. We can now identify a venous interperiosteodural space connecting (black arrowheads) the SS with the IPS inferior to CNs X and XI. B: Superomedial view of the same right JF as in panel A after petrosal drilling and removal of the intrajugular process, the dural sheath of CN IX, and the intraluminal blue latex, showing the intraforaminal dura and the EL of the roof of the jugular bulb (JB). Due to the high position of the dome of the jugular bulb, it may be an obstacle to some surgical procedures. 

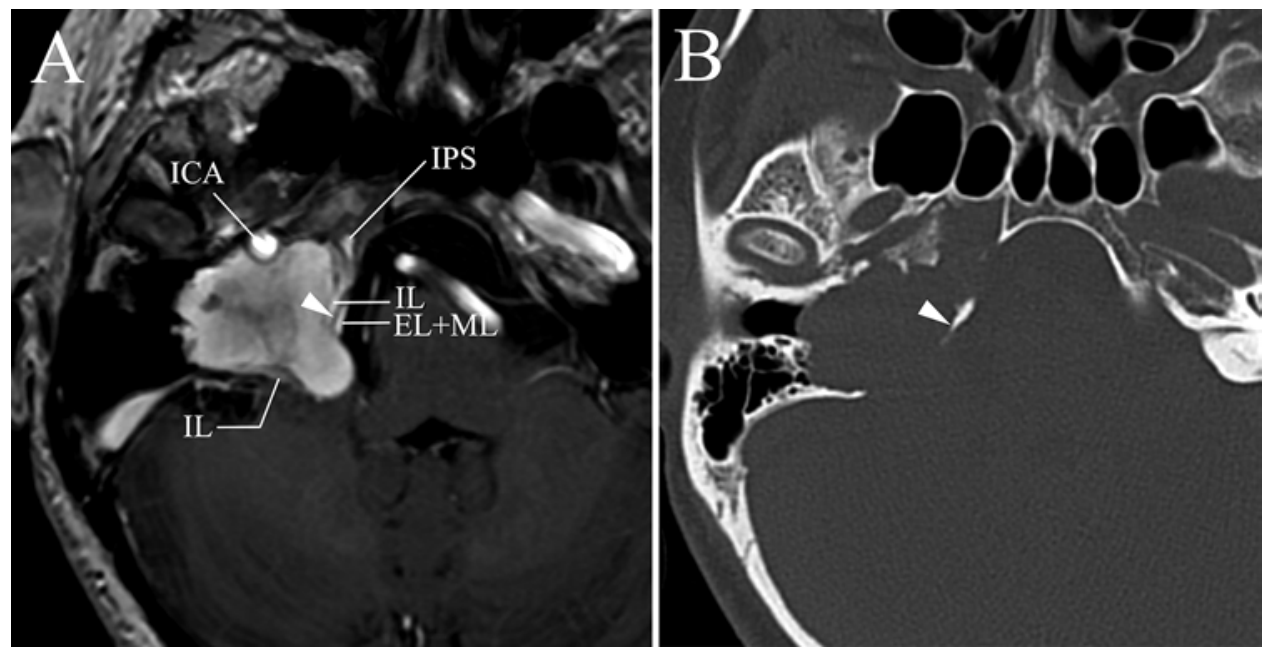

FIG. 7. Case 1. Images obtained in a 37-year-old woman with a lower cranial nerve neurofibroma A: Axial contrast-enhanced T1-weighted MR image showing a dumbbell-shaped enhancing tumor of the JF, which is pushing the IPS anteriorly. The vertical petrous segment of the ICA is not reached by the tumor. The jugular bulb, narrowed by the tumor, is not visible. At the anterior aspect of the tumor, the intracranial dura mater, formed by both the EL and $\mathrm{ML}$, is identified as a thin line of contrast enhancement. Inside the JF, at the anterior and posterior aspects of the tumor, the anterior and posterior components of the IL are identifiable. The anterior component of the IL is separated from the intracranial dura mater by a small ridge of cortical occipital bone (white arrowhead). B: Axial CT image showing a mass lesion that developed inside the right JF causing a scalloping of the petrous and occipital bones. The intrajugular ridge has been lysed by the tumor and is not visible. A thin cortical band of the occipital bone has been preserved (white arrowhead).

We propose to call these 2 thickenings, in combination, the "intrajugular ligament" (IL).

The inferior wall of the horizontal part of the dural sheath of CN IX, as well as the superior wall of the horizontal part of the dural sheath of CNs X and XI, did not enter into contact with bone (Figs. 1B and 2A). We can conclude that they were composed of ML only. In contrast, the lateral wall of the dural sheath of the vertical part of CN IX and the medial wall of the dural sheath of the vertical part of CNs X and XI were in contact with the petrous bone (into the pyramidal fossa). These dural sheaths were then, at least in part, composed of EL. Nonetheless, it was impossible to ascertain macroscopically whether the ML extended to this level.

The confluence of the IPS and the jugular bulb was located in the interperiosteodural space. This interperiosteodural communication between the IPS and the SS was exposed after opening the ML and partially removing intraluminal content (blue latex) (Fig. 4A). This confluence of the IPS and the jugular bulb was found between the sheath of CN IX and the sheath of CNs X and XI, where these nerves were turning into the JF (Figs. 1B, 2A, and 4A). We conclude that the most common venous communication (16 specimens, $80 \%$ ) is located between the sheaths of CN IX and CNs X-XI.

\section{Illustrative Cases}

\section{Case 1}

A 37-year-old woman with neurofibromatosis Type 1 was initially admitted to our institution in 2013 for a right facial palsy that had been progressively increasing for several months. She had also observed gradually increasing ataxia, right hypoacousis, and difficulty swallowing during the previous month. Clinical examination showed HouseBrackmann Grade VI facial palsy, right cophosis, and lower cranial nerve palsy with swallowing disturbances. CT and MRI revealed a contrast-enhancing JF lesion with scalloping of the intrajugular ridge (Fig. 7B). On axial MR images, the lesion appeared triangular and could be seen to have pushed the IPS anteriorly (Fig. 7A).

Extensive examination of radiological data revealed some of the meningeal layers surrounding the intraforaminal part of the tumor. At the anterior aspect of the tumor, the intracranial dura mater, formed by both the EL and ML, is identifiable as a thin linear contrast enhancement. Inside the JF, we assumed that, at the anterior and posterior aspects of the tumor, the components of the IL were identifiable. The anterior component of the IL is separated from the intracranial dura mater by a small ridge of cortical occipital bone (Fig. 7A, white arrowhead). However, because of the tumor's mass effect, the axial MR image does not show the anterior and posterior components of the IL reaching up to a single anterior point, but instead as separated from each other.

During the operation performed on this patient, both translabyrinthine and retrosigmoid approaches were used to gain access to the tumor. Piecemeal subtotal removal of the tumor was achieved; CN XI was involved in most of the lesion. Intraoperatively, due to the deformation of anatomical structures by the tumor, it was impossible to ascertain macroscopically which layer extended to this level. After surgery, the patient had a transient swallowing disorder. Histopathological examination of the resected tissue demonstrated neurofibroma. The patient's cophosis and facial nerve palsy remained stable for the 3 years of follow-up. 

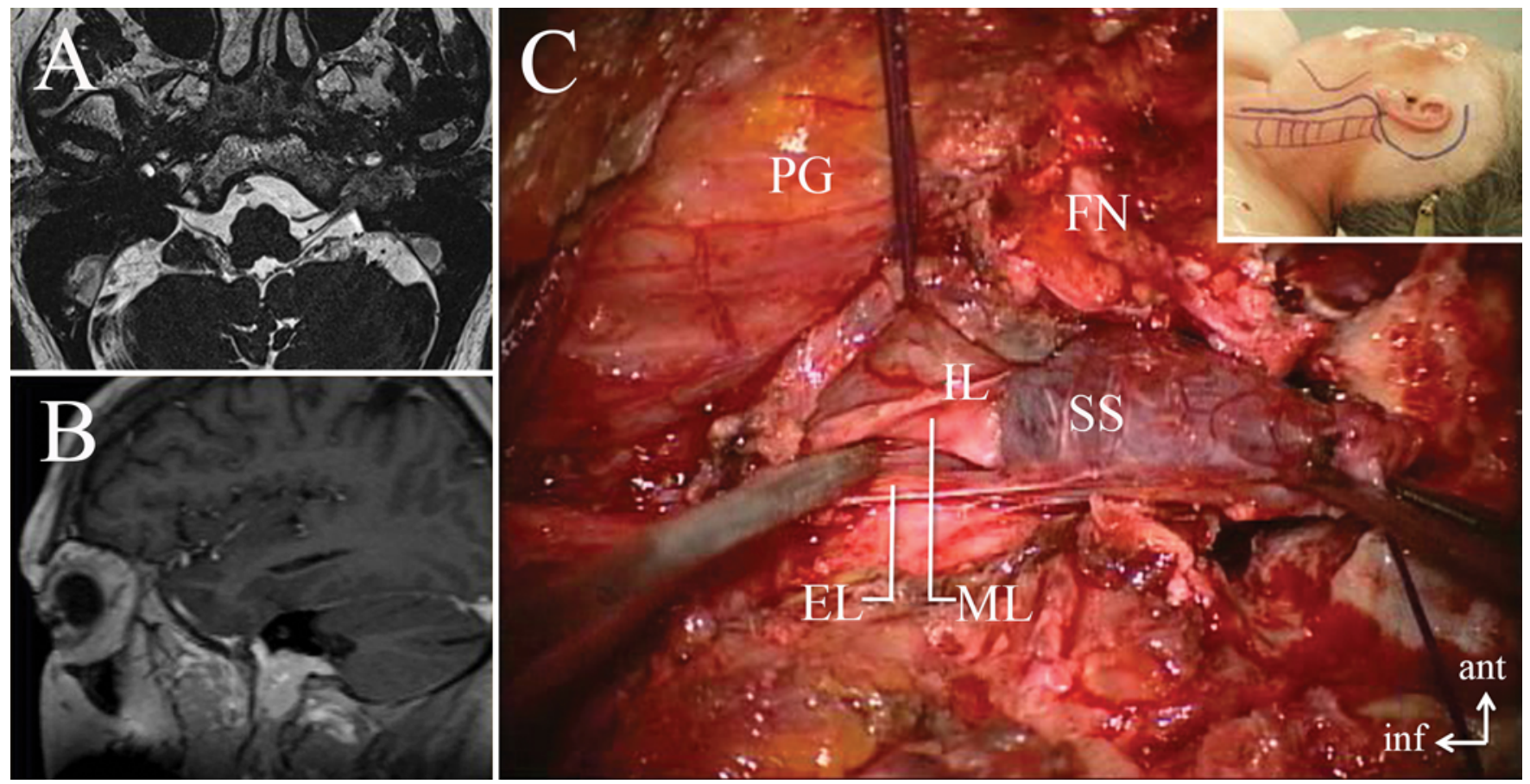

FIG. 8. Case 2. Surgical case of a 45-year-old woman with a history of neck pain associated with hypacousis and some episodes of dysphagia. A and B: Axial T2-weighted CISS (constructive interference in steady state) (A) and sagittal T1-weighted gadolinium enhanced (B) MR images obtained on admission demonstrating a large mass occupying the left JF with extension to the neck. C: Intraoperative photograph. The patient underwent surgical removal of the mass via an infralabyrinthine approach with rerouting of the facial nerve after SS embolization. After jugular vein ligature and opening of the EL of the SS, the tumor was resected. Tumor involvement of the thrombosed SS allowed the surgeon to open the EL of the SS and remove the tumor without major bleeding. The IL (a thickening of the $\mathrm{ML}$ ) protects the lower cranial nerves, serving as a surgical border that allows optimal tumor removal without lower cranial nerve deficits. FN = facial nerve; inf = inferior; $M L=$ meningeal layer of CNs IX-XI; PG = parotid gland.

\section{Case 2}

This 45-year-old woman presented with neck pain associated with hearing loss and some episodes of dysphagia. MRI and CT examinations depicted a large mass occupying the JF with extension to the neck (Fig. 8A and B). Angiography showed a highly vascularized mass fed by the ICA and external carotid artery and its branches. Tumor feeders and the sigmoid sinus were obstructed preoperatively by endovascular embolization. The patient underwent surgical removal of the mass via an infralabyrinthine approach with rerouting of the facial nerve (Fig. 8C). After jugular vein ligature and opening of the EL of the SS, a complete resection was performed. During this procedure, the IL is a natural surgical border inside the JF, protecting the lower cranial nerves. Following the operation, the patient experienced a transient mild facial palsy, which resolved completely. The histopathological findings were consistent with a paraganglioma. After 8 years of follow-up, MRI demonstrated no evidence of any residual or recurrent lesion.

\section{Discussion}

In this study, we provide an extensive description of the anatomy of the JF and its dura mater. The complexity of this anatomy is partly due to the existence of different dural layers, which separate at the level of the JF, forming an interperiosteodural space. The existence of an interperi- osteodural space inside the JF encourages us to propose a novel compartmentalization of the JF.

\section{Interperiosteodural Space}

The interperiosteodural concept, which has been called the EDNAC since Parkinson's description, ${ }^{12}$ is an anatomical complex that extends from the coccyx to the orbit. It contains valveless veins and sometimes adipose tissue. In our study, the medial wall of the JF, i.e., its endocranial wall, was formed by the ML, while the floor and roof of the JF were formed by the EL. Between these layers was found an interperiosteodural space within which the SS and IPS coursed.

The ML, which covered the petro-occipital fissure, formed an arch within which passed CNs X and XI. This dural fold was comparable to the falciform ligament of the optic nerve. In all specimens, the anterior part of the ML of the glossopharyngeal porus and the posterior part of the ML of the vagal porus formed an intrajugular ligament (IL) similar to the Gruber ligament and directed upward and anteriorly in the direction of the porus of the facial nerve.

Identification of the dural sheaths of lower cranial nerves is challenging. In our study, the lateral wall of the dural sheath of the vertical part of CN IX was in contact with the petrous bone, and thus this dural sheath was at least partially made of EL. Nonetheless, it was impos- 
sible to determine macroscopically whether the ML was extending to this level and may also have made up part of the dural sheath. However, the presence of ML inside the lateral wall of the dural sheath of the vertical part of CN IX is conceivable, as the ML formed the medial part of this sheath. The medial wall of the dural sheath of the vertical part of CNs X and XI was in contact with the occipital bone (into the pyramidal fossa). Therefore, this dural sheath was made at least partially of EL. But macroscopically, as for CN IX, it was impossible to determine whether the ML was extending to this and may also have made up part of the dural sheath. However, as the ML formed the lateral part of this sheath, the presence of the ML inside the medial wall of the dural sheath of the vertical part of CNs X and XI is conceivable.

Finally, in 4 specimens we observed an interperiosteodural space, where the connection between the SS and IPS was located, that was inferior to the CN X-XI dural sheath. The relative positions of the $\mathrm{CN}$ X-XI dural sheath and this interperiosteodural space are similar to those of the pituitary gland and the coronary sinuses located beneath it. ${ }^{4}$ Likewise the pituitary gland, $\mathrm{CN} \mathrm{X}$, and $\mathrm{CN} \mathrm{XI}$ were embedded in a dural sac. For the JF, however, this communication did not join 2 similar sinuses in the midline. The communication between the SS and IPS was located in these specimens between this dural sac and the occipital bone.

\section{JF Compartmentalization}

JF compartmentalization is controversial. Indeed, the first 2-compartment classification of the JF is generally credited to Hovelacque. ${ }^{9}$ According to this classification, the anteromedial pars nervosa contains the CN IX and the IPS, and the posterolateral pars venosa contains CNs $\mathrm{X}$ and XI, the jugular bulb, and the posterior meningeal artery. Shapiro ${ }^{17}$ described another 2-part compartmentalization, based on osteology and radiographic study. $\mathrm{He}$ showed that the anteromedial part contained the IPS and CNs IX, X, and XI and was anterior to the intrajugular petrous ridge. The jugular bulb and the posterior meningeal artery, which are posterior to the bony intrajugular ridge, formed the posterolateral part. This compartmentalization does not seem to be pertinent in an anatomosurgical field because of the huge diameter of the IPS. Moreover, the importance of the venous space and the connection between the IPS and SS are not in accordance with the concept of a single pars venosa containing venous blood.

In their studies of the microsurgical anatomy of the JF, neither Kveton and Cooper ${ }^{11}$ nor Ayeni et al..$^{2}$ found any instance of discrete compartmentalization within the foramen. According to Ayeni et al., an endocranial division exists between CN IX and CNs X-XI, but this division is not found intraforaminally. They studied the JF from both the infratemporal fossa approach and the dorsolateral approach, after the SS and jugular bulb were opened, and showed that the lower cranial nerves lay in "a loose sheath of connective tissue and were all anteromedial to the SJB [superior jugular bulb], with CN IX being the most anterior and CN XI the most posterior." This connective tissue described by Ayeni and colleagues corresponds to our description of the ML of CN IX and CNs X-XI. Moreover, these authors described the intraforaminal part of CN IX as slightly lateral to CNs X and XI. They thought that the differences in the relationships of the lower cranial nerves between the endocranial and intraforaminal parts of the JF indicated that no compartmentalization was possible. In contrast, our study of dural layers shows that the separation between CN IX and CNs X-XI is present in both the endocranial and the intraforaminal parts of the JF.

A more recent dural septation description, based on microsurgical anatomy, from Katsuta et al., ${ }^{9}$ has divided the JF into 3 compartments: a large posterolateral venous channel (called the sigmoid part) that receives the flow of the SS, a small anteromedial venous channel that receives the drainage of the IPS (called the petrous part), and an intermediary neural compartments (called the neural compartment), which is located between the sigmoid and the petrosal parts. According to Katsuta et al., the neural part is an intrajugular part through which the lower cranial nerves course. Nevertheless, our analysis shows that $\mathrm{CN}$ IX is separated most frequently from CNs X and XI by an interperiosteodural space containing the posterior end of the IPS, which drains into the SS. Moreover, we describe an anatomical division of the nervous compartment, which is composed of 2 porus and 2 dural sheaths for, respectively, CN IX and CNs X-XI.

Our study of the dural layers permits us to describe an original 3-part compartmentalization of the JF: 1) the glossopharyngeal compartment, which contains CN IX coursing in its own dural sheath; 2) the vagal compartment, which contains CNs $\mathrm{X}$ and XI, coursing in a common dural sheath; and 3) the interperiosteodural compartment, which includes the interperiosteodural space between EL and ML, which contains venous blood of the IPS and the $\mathrm{SS}$, and the communication between these 2 sinuses.

\section{Surgical Implications}

The communication between the IPS and SS is most frequently located between the inferior sheath of CN IX (ML) and the superior sheath of CNs X and XI (ML). The anterior part of the IL could be understood as a thick meningeal ligament anterior to CN IX. Besides, the IPS is found ventral to the porus of CN IX. Hence, due to the existence of the IL, which protects the porus of these lower cranial nerves, drilling of the petrous bone at the anterior part of the JF can be considered safe when it is performed anteriorly and superiorly to CN IX. The anatomosurgical concept of a posterior pars venosa and an anterior pars nervosa (allowing an anterior petrous drilling) is, therefore, according to our surgical experience and the results of our study, not very pertinent anatomically. Another key point is that when exiting the JF, CN IX is tethered to the ICA by a small carotid ridge. Because of this proximity, and also because of its anterior position, CN IX may be the most difficult cranial nerve to identify during upper cervical and dorsolateral posterior fossa surgery and the most vulnerable to iatrogenic injury. ${ }^{2,21}$

Considering paraganglioma surgery and lateral skull base approaches (petrosal approaches, such as Fisch Type $\mathrm{A}$, and retrolabyrinthine transcochlear approaches), tumor involvement of the SS, which is frequently thrombosed, allows the surgeon to open the EL of the SS (after liga- 
ture of the internal jugular vein). During this procedure, the IL (a thickening of the ML) protects the lower cranial nerves. The IL is then a surgical border that allows optimal tumor removal without lower cranial nerve deficits. Nevertheless, as these tumors can invade the carotid artery, the surgeon should always keep in mind that the EL and carotid ridge could have paraganglioma involvement and therefore should not be considered to be consistent surgical landmarks.

Finally, extensive drilling of the posterolateral half of the occipital condyle in far-lateral, extradural anterolateral, and extreme-lateral approaches can classically induce hypoglossal nerve deficit. ${ }^{1,3,5,6,10,16}$ But one should keep in mind that the other lower cranial nerves are close and may also be injured during these approaches.

\section{Conclusions}

Our study focused on the anatomical meningeal features of the JF. The structures that traverse the JF are the SS and IPS; jugular bulb; CNs IX, X, and XI; and the meningeal branches of the ascending pharyngeal and occipital arteries. Over the past 70 years, the JF has been well described using microsurgical dissection in cadavers. However, we present the first description focusing especially on the meningeal architecture of the JF. This original anatomical approach allowed us to demonstrate the inaccuracy of the classical compartmentalization of the JF into the so-called pars nervosa and pars venosa and to propose a novel compartmentalization into 3 parts: 1) the glossopharyngeal compartment, which includes CN IX coursing in its own dural sheath; 2) the vagal compartment, which includes CNs X and XI coursing in a common dural sheath; and 3) the interperiosteodural compartment through which passes the venous blood of the IPS and the SS, and which provides communication between these 2 sinuses.

\section{Acknowledgments}

We are indebted to Gerald Deluermoz, Jean Paul Da Silva (Université François Rabelais de Tours, Laboratoire d'Anatomie), Daniel Bourry (Université François Rabelais de Tours, Service d'Iconographie), and Pr. Laurent Laccoureye (CHU d'Angers, Otorhinolaryngology Department) for their technical help.

\section{References}

1. Açikbaş SC, Tuncer R, Demirez I, Rahat O, Kazan S, Sindel M, et al: The effect of condylectomy on extreme lateral transcondylar approach to the anterior foramen magnum. Acta Neurochir (Wien) 139:546-550, 1997

2. Ayeni SA, Ohata K, Tanaka K, Hakuba A: The microsurgical anatomy of the jugular foramen. J Neurosurg 83:903-909, 1995

3. Babu RP, Sekhar LN, Wright DC: Extreme lateral transcondylar approach: technical improvements and lessons learned. J Neurosurg 81:49-59, 1994

4. Destrieux C, Kakou MK, Velut S, Lefrancq T, Jan M: Microanatomy of the hypophyseal fossa boundaries. J Neurosurg 88:743-752, 1998

5. Destrieux C, Velut S, Kakou MK, Lefrancq T, Arbeille B, Santini JJ: A new concept in Dorello's canal microanatomy: the petroclival venous confluence. J Neurosurg 87:67-72, 1997
6. Dowd GC, Zeiller S, Awasthi D: Far lateral transcondylar approach: dimensional anatomy. Neurosurgery 45:95-100, 1999

7. François P, Travers N, Lescanne E, Arbeille B, Jan M, Velut $\mathrm{S}$ : The interperiosteo-dural concept applied to the perisellar compartment: a microanatomical and electron microscopic study. J Neurosurg 113:1045-1052, 2010

8. François P, Zemmoura I, Fouquet AM, Jan M, Velut S: Lateral sellar angiolipoma: a tumor illustrative of the extradural compartment of the neural axis. J Neurosurg 113:10531058,2010

9. Katsuta T, Rhoton AL Jr, Matsushima T: The jugular foramen: microsurgical anatomy and operative approaches. Neurosurgery 41:149-202, 1997

10. Kratimenos GP, Crockard HA: The far lateral approach for ventrally placed foramen magnum and upper cervical spine tumours. Br J Neurosurg 7:129-140, 1993

11. Kveton JF, Cooper MH: Microsurgical anatomy of the jugular foramen region. Am J Otol 9:109-112, 1988

12. Parkinson D: Extradural neural axis compartment. J Neurosurg 92:585-588, 2000

13. Parkinson D: History of the extradural neural axis compartment. Surg Neurol 54:422-431, 2000

14. Rhoton AL Jr: Jugular foramen. Neurosurgery 47 (3 Suppl):S267-S285, 2000

15. Rhoton AL Jr, Buza R: Microsurgical anatomy of the jugular foramen. J Neurosurg 42:541-550, 1975

16. Salas E, Sekhar LN, Ziyal IM, Caputy AJ, Wright DC: Variations of the extreme-lateral craniocervical approach: anatomical study and clinical analysis of 69 patients. J Neurosurg 90 (2 Suppl):206-219, 1999

17. Shapiro R: Compartmentation of the jugular foramen. J Neurosurg 36:340-343, 1972

18. Taptas JN: La loge du sinus caverneux; sa constitution et les rapports des éléments vasculaires et nerveux qui la traversent. Sem Hop 25:1719-1722, 1949

19. Taptas JN: [The parasellar osteo-dural chamber and the vascular and neural elements that traverse it. An anatomical concept that would replace the cavernous sinus of classical anatomy.] Neurochirurgie 36:201-208, 1990 (Fr)

20. Taptas JN: The so-called cavernous sinus: a review of the controversy and its implications for neurosurgeons. Neurosurgery 11:712-717, 1982

21. Tummala RP, Coscarella E, Morcos JJ: Surgical anatomy of the jugular foramen. Oper Tech Neurosurg 8:2-5, 2005

\section{Disclosures}

The authors report no conflict of interest concerning the materials or methods used in this study or the findings specified in this paper.

\section{Author Contributions}

Conception and design: Bernard, Velut. Acquisition of data: Bernard, Fournier. Analysis and interpretation of data: Bernard, Cottier. Critically revising the article: Bernard, Zemmoura, Cottier, Terrier, Velut. Reviewed submitted version of manuscript: Zemmoura, Terrier, Velut. Approved the final version of the manuscript on behalf of all authors: Bernard. Administrative/technical/ material support: Fournier. Study supervision: Velut.

\section{Correspondence}

Florian Bernard, Service de Neurochirurgie, CHU, 4 rue Larrey, Angers 49100, France. email: bernardflorian.bf@gmail.com. 